CAHIERS DE

NARRATOLOGIE

\section{Cahiers de Narratologie}

Analyse et théorie narratives

31 Bis | 2017

Espace du récit, récit de l'espace en contexte germanique

\title{
Espace du texte et énoncés généralisants en ouverture de textes narratifs littéraires ou narration et performance dans les récits de fiction
}

\section{Anne-Laure Daux-Combaudon}

\section{OpenEdition}

\section{Journals}

Electronic version

URL: http://journals.openedition.org/narratologie/7733

DOI: 10.4000/narratologie. 7733

ISSN: $1765-307 X$

Publisher

LIRCES

Electronic reference

Anne-Laure Daux-Combaudon, « Espace du texte et énoncés généralisants en ouverture de textes narratifs littéraires ou narration et performance dans les récits de fiction », Cahiers de Narratologie [Online], 31 Bis | 2017, Online since 26 June 2017, connection on 19 April 2019. URL : http:// journals.openedition.org/narratologie/7733; DOI : 10.4000/narratologie.7733

This text was automatically generated on 19 April 2019

Article L.111-1 du Code de la propriété intellectuelle. 


\title{
Espace du texte et énoncés généralisants en ouverture de textes narratifs littéraires ou narration et performance dans les récits de fiction
}

\author{
Anne-Laure Daux-Combaudon
}

\section{Introduction}

1 Même si cela ne constitue pas l'élément le plus évident pour le texte narratif littéraire, celui-ci se définit aussi par sa matérialité ${ }^{1}$. Il est courant aujourd'hui dans la tradition allemande de la linguistique textuelle [Textlinguistik] d'ajouter aux sept critères définitoires de la textualité établis par Robert Beaugrande et Wolfgang Dressler ${ }^{2}$ cinq critères «non-verbaux » présentés par Ulla Fix, à savoir non seulement les phénomènes de pensée intrinsèques à la langue que sont l'unité de la forme et la culturalité mais aussi les phénomènes extra-linguistiques concrets et palpables d'apparition du texte: sa médialité, sa matérialité et sa localité3. Dans cette perspective, on n'approche pas les textes écrits seulement ou en premier lieu comme des signes linguistiques complexes articulant un ensemble de sens, ce sont aussi des images ${ }^{4}$, des dessins reçus visuellement dans leur bi-dimensionalité ${ }^{5}$, des surfaces visuelles ${ }^{6}$, des espaces de texte - dans lesquels interviennent des phénomènes de nature non exclusivement verbale. Dans la tradition française de la linguistique textuelle, cette question est abordée par Jean-Michel Adam lorsqu'il évoque le « régime de matérialité » d'un texte ${ }^{7}$ ou lorsqu'il introduit la notion de segment, définie comme « unité vi-lisible chargée, en fait, de souligner un PLAN DE TEXTE » 8.

2 Qu'appelle-t-on la matérialité d'un texte? C'est la mise en forme de son support. Pour l'oral $^{9}$ cela touche à la performance au sens de la rhétorique, pour l'écrit surtout à la 
typographie et à la surface visuelle ${ }^{10}$. Selon Harmut Stöckl, la matérialité d'un texte écrit, i.e. ses dispositifs typographiques, compte aussi, à côté des niveaux propositionnel, illocutoire et locutoire, parmi les caractéristiques à même de nous faire reconnaître un type de texte, d'autant plus que dans la réception, la matérialité est saisie conjointement voire antérieurement aux structures linguistiques ${ }^{11}$.

Ainsi un texte narratif littéraire se reconnaît dès son début ${ }^{12}$, à sa manière d'occuper la page blanche, comme en témoignent les illustrations 1 et 2 . Le texte narratif commence après les paratextes éditoriaux (couverture, page de titre) et auctoriaux (dédicace, préface). Pour les romans et les autobiographies, le récit commence sur la page de droite, en haut, mais plus bas que la ligne supérieure (marquée éventuellement par un titre de chapitre ou un numéro, mais aussi sans aucun indicateur autre que la position sur la page). Pour les nouvelles, le récit peut commencer à droite ou à gauche et, selon les éditeurs, on commencera toute nouvelle avec une nouvelle page ou on enchaînera toutes les nouvelles les unes à la suite des autres après un blanc sur la page.

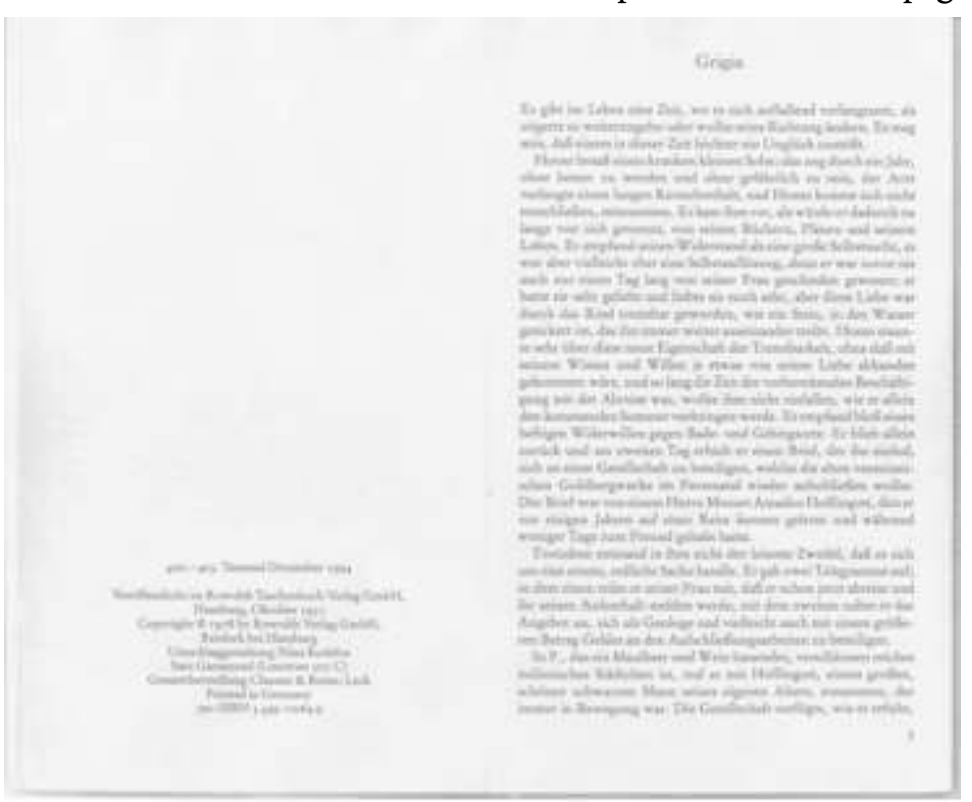

Illustration 1 : Photographie du début d'une nouvelle de Robert Musil, « Grigia » [Grigia] [1923], dans id., Drei Frauen, Reinbek bei Hambourg, 1978, p. 5-25. 


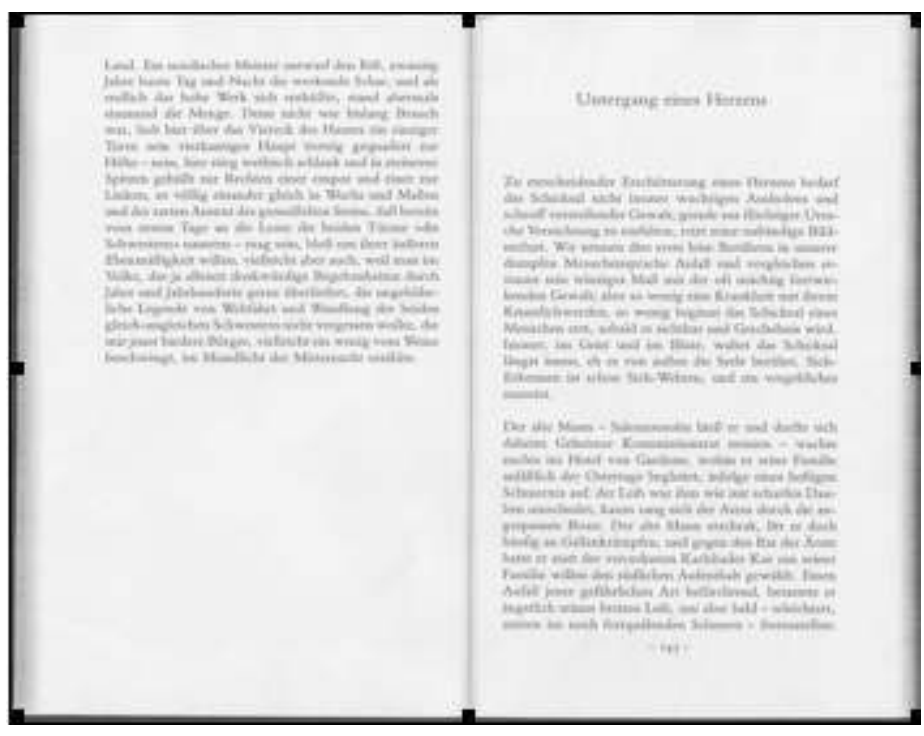

ILLUSTRATION 2 : PHOTOgRAPHIE DU DÉbUt d'UNE NOUVELLE DE STEFAN ZWEIg, « UNTERgANg EINES HERZENS " [NAUFRAgE D'UN COEUR] [1926], DANS ID., VERWIRRUNg DER GEFÜHLE, ERZÄHLUNgEN, FRANCFORT-SUR-LE-MAIN, 1984, P. 145-181.

4 Et en général, le début du texte narratif littéraire se reconnaît aussi à son contenu thématique. Pour Jean-Michel Adam, la séquence narrative s'ouvre sur une phase d'orientation-exposition ${ }^{13}$ qui apporte des informations et désigne les circonstances du monde (temps et localisation) ou les composantes (acteurs, monde et action ou événement ${ }^{14}$ ). Par ailleurs, on peut aussi trouver au début de textes narratifs des « entrées-préfaces » ou « résumés », se situant à un autre niveau que le récit, qui servent «à permettre le passage de la conversation au récit, [et] surtout à imposer la prise de parole narrative ». Il faut y voir, selon lui, des marques de « passage de la conversation au récit $»^{15}$. L'illustration 3 ci-dessous rend compte de cette présentation:

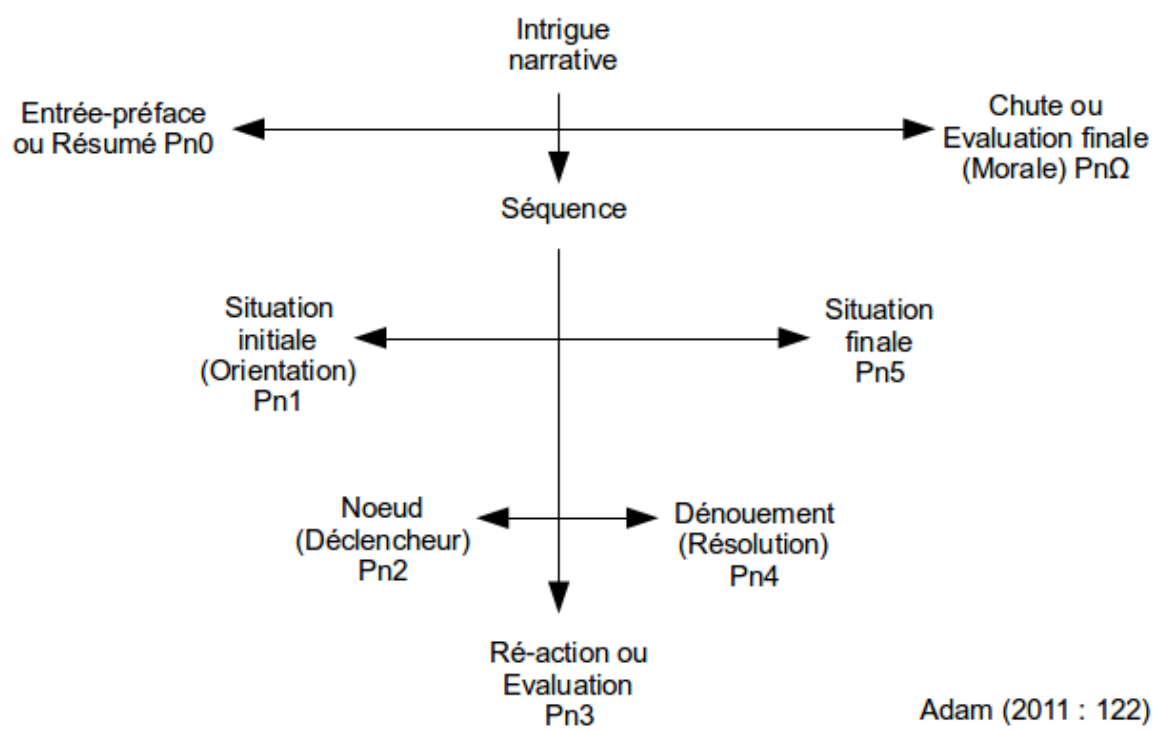

Illustration 3 : la séquence narrative selon Adam, 2011 (note 14), p. 122. 
Pour Annette Retsch, les débuts s'ouvrant sur des entrées-préfaces constituent, pour les textes narratifs littéraires, un certain type de début parmi d'autres. Ainsi, elle distingue trois types de débuts narratifs:

6 - le début de texte ab-ovo qui se caractérise au niveau thématique par des informations sur le lieu, le temps, les caractères de personnages

- le début in-medias-res qui plonge le lecteur au milieu de l'histoire

- le début de texte métatextuel, dans lequel l'auteur commente les événements, les faits ou le récit lui-même ${ }^{16}$.

7 Pour ces trois types de début, il est évidemment nécessaire de considérer qu'il y a des différences selon le contexte d'écriture, que ces différences peuvent correspondre à des phénomènes de mode littéraire, ou à des évolutions selon les siècles.

8 Selon Retsch, le début métatextuel par exemple est le résultat d'une évolution du paratexte, et plus précisément de la perte de popularité de la préface au xIx ${ }^{\text {ème }}$ siècle :

Le début métatextuel est à mon avis aussi à considérer comme la conséquence de l'évolution du paratexte. Comme la rédaction de préface perd en popularité au XIX ème siècle, les auteurs comme Raabe et Frenssen choisissent à la fin du XIx ${ }^{\text {ème }}$ siècle et au début $\mathrm{du} x \mathrm{xx}^{\mathrm{eme}}$ siècle le lieu destiné au début de texte pour communiquer certaines intentions ${ }^{17}$.

9 Nous voulons nous intéresser ici à une catégorie de débuts dits métatextuels, à savoir les débuts de texte narratif littéraire marqués par un énoncé généralisant. Ce sont des énoncés qui ont pour point commun de ne pas relever du récit (erzählte Zeit) mais du niveau du discours (erzählende Zeit). Ils peuvent donner lieu ou non à une segmentation de l'espace du texte. Ils correspondent matériellement sur la page à un segment textuel à part ou à un paragraphe à part, ou encore ils sont associés au reste du corps du texte, sans que ces différences ne soient systématiquement liées aux phénomènes d'anaphore entre l'énoncé généralisant et la suite du texte, ce dont témoignent les extraits 1 à 4 .

10 En outre, ces débuts sont remarquables aussi parce qu'à l'énoncé généralisant peut faire suite un début narratif relevant d'un des deux autres types de début narratif définis par Annette Retsch. On trouve après l'énoncé généralisant par exemple un début ab-ovo donnant des informations sur le temps (extrait 5), sur le lieu (extraits 5 et 6 ) ou sur un personnage (extraits 7 et 8), ou encore un début in-medias-res (extrait 9).

11 L'analyse des énoncés généralisants en ouverture de textes narratifs littéraires conduit donc à interroger la notion de début du texte narratif littéraire. Quand le récit commence-t-il véritablement? Quel est le vrai début du texte narratif: son début matériel ou son début thématique ? Quelle(s) fonction(s) remplit l'énoncé généralisant en ouverture de textes narratifs littéraires?

12 Afin de répondre à ces questions, nous développerons dans un premier temps une définition de l'objet d'étude "énoncé généralisant » avant de détailler dans un second temps les fonctions textuelles que remplissent les énoncés généralisants en ouverture de textes narratifs littéraires. Nous montrerons enfin que ce phénomène textuel illustre de manière radicale que le récit de fiction se caractérise par un double niveau de fiction et que la fiction de la narration ne constitue pas un « supplément » du récit. 


\section{Définition de l'objet d'étude « énoncé généralisant »}

13 L'énoncé généralisant est une notion que nous avons été amenée à définir en raison de leur présence dans certaines autobiographies est-allemandes d'après $1989^{18}$. C'est alors plutôt leur rôle dans le corps du texte qui a été expliqué, les énoncés généralisants s'apparentant à une forme de reformulation, de retour sur le dire, qui sert la compréhension de ce qui vient d'être dit/écrit.

14 Par différence avec le travail de Jean-Christophe Pellat sur les Maximes de La Rochefoucauld, qui insiste sur le fait que ses maximes "se coulent dans les moules linguistiques de la généralité » en même temps qu'elles "portent des marques de la présence » de l'auteur et qu'elles se caractérisent donc par une «tension entre une visée générale et une intention individuelle ${ }^{19}$, nous voyons dans la généralité non pas un discours sans sujet, mais plutôt un discours de tous les sujets : il n'y a pas pour nous de contradiction entre marques de subjectivité et généralisation. L'énoncé généralisant joue un rôle déterminant parmi les phénomènes d'ajustement intersubjectifs ${ }^{20}$ et doit être considéré comme un indice de dialogisme ${ }^{21}$.

15 Reprenant la définition de Magid Ali Bouacha, ${ }^{22}$ nous définissons l'énoncé généralisant comme un énoncé toujours vrai reposant sur une opération de parcours ${ }^{23}$. Les deux critères décisifs sont donc la dimension énonciative de l'énoncé et l'opération de parcours à laquelle il donne lieu.

16 Du point de vue énonciatif, un énoncé généralisant exprime une vérité générale : c'est un énoncé qui peut être répété. Le phénomène se laisse bien expliquer par la théorie de la polyphonie. Les premiers travaux sur la polyphonie sont habituellement attribués à Oswald Ducrot. La théorie de la polyphonie refuse le postulat de l'unicité du sujet parlant. En polyphonie, « le sens d'un discours est fondamentalement constitué par un faisceau de discours qui se manifestent en lui $»^{24}$. Toute énonciation comporte ainsi l'expression d'une pluralité de voix constitutives de son sens, parmi lesquelles :

17 - le sujet parlant (ou auteur empirique de l'énoncé)

- le locuteur (ou auteur désigné de l'énoncé)

- les énonciateurs (ou rôles discursifs mis en scène par le locuteur)

18 Lorsque les énonciateurs se confondent avec l'opinion générale ou l'opinion publique, dénotant une doxa anonyme, on peut parler de «ON-vérité» ${ }^{25}$. Pour Jean-Claude Anscombre, il faut voir notre savoir comme représenté par différents ON-locuteurs ou par différentes communautés linguistiques ${ }^{26}$. Le locuteur peut évoquer ces différents types de savoir. Derrière ON, on peut donc trouver l'opinion publique, une rumeur, une loi scientifique ou un groupe social donné. Mais attention : parler de ON-locuteur n'implique pas nécessairement la présence du pronom " on » / man dans l'énoncé.

19 C'est cette particularité énonciative des énoncés généralisants qui fait qu'ils peuvent être répétés. S'ils peuvent être répétés, cela signifie aussi qu'ils ont déjà été dits une fois au moins. C'est particulièrement vrai pour les proverbes ou pour les lois scientifiques dont on peut en principe déterminer la première formulation. Dans tous les cas, c'est bien le groupe de tous les locuteurs potentiels de cet énoncé qui lui confère sa dimension de vérité générale. 
20 Ce critère définitoire énonciatif explique la dimension polyphonique de ces énoncés généralisants. Lorsqu'ils sont utilisés dans le corps du texte narratif, ils constituent une rupture énonciative ${ }^{27}$. Le narrateur quitte le niveau du récit pour le niveau du discours.

21 Le deuxième critère définitoire de l'énoncé généralisant touche à l'opération de parcours qu'il déclenche à travers toute une classe. Les énoncés généralisants s'avèrent valides pour une multitude de situations. Ils ne sont pas valides pour une situation particulière mais bien pour une classe de situations. Ainsi, dans l'extrait 1 c'est l'ensemble de la classe des cœurs humains qui est considérée avant de s'arrêter sur le personnage de Salomonsohn; en 2 c'est à la classe des individus qu'il est fait référence avec «tout homme "; en 3 le curseur parcourt la classe des amateurs-nés; en 4 enfin la classe des petits manques. Introduits dans le corps du texte narratif, ils fonctionnent comme des moules interprétatifs pour une situation particulière ${ }^{28}$. En ce sens, ils participent de l'intersubjectivité.

Deux remarques s'imposent après cette définition. D'abord, cette définition des énoncés généralisants inclut les énoncés génériques mais aussi des énoncés contenant des indicateurs spatio-temporels ou des opérateurs modaux tels que " pouvoir ». Ali Bouacha indique ainsi que "plus les énoncés sont ancrés dans le discours et plus ils ont des chances d'être acceptés comme énoncés généralisants ${ }^{29}$. C'est bien ainsi que fonctionnent les extraits 11 et 12 .

23 Ensuite, cette définition de l'énoncé généralisant implique la présence possible d'adresses aux interlocuteurs, i.e. lecteurs, et de modalisateurs (voir l'extrait 4).

24 Les indices linguistiques permettant l'identification d'un énoncé généralisant ont été plusieurs fois relevés. Il s'agit principalement de l'indéfinitude, marquée en allemand par le pronom personnel man (extraits 3 ou 5), par l'article indéfini ein (extraits 6 et 7), par les quantificateurs indéfinis (extrait 8), par les quantificateurs globaux ${ }^{30}$ (extraits 9 et 10), par les W-Wörter (extrait 11), il s'agit aussi du présent de vérité générale (extraits 3, 5 et 6).

25 Les énoncés généralisants sont donc bien des énoncés qui se situent au niveau de la narration, au niveau de l'interaction entre le narrateur et le lecteur. Constitutivement, donc, ce ne sont pas des éléments du récit à proprement parler, ils ne donnent pas de premiers éléments d'information thématique.

Indices très nets d'un décrochement énonciatif quand ils sont utilisés dans le corps du texte, on passe, avec les énoncés généralisants, du récit au temps du discours (erzählte Zeit vs. erzählende Zeit). Ils constituent donc un lieu où le narrateur tient compte soit de l'opinion de son lecteur soit des connaissances de celui-ci pour ajuster son discours à celles-ci. C'est aussi un lieu où le lecteur doit fournir un certain travail interprétatif, travail de coopération pour co-construire le sens du texte qui lui est donné.

27 Mais ces énoncés généralisants apparaissent aussi en ouverture de texte narratif littéraire. Quelle est alors leur fonction ? Retardent-ils le véritable début de l'histoire?

\section{Le corpus}

28 Afin de répondre à ces questions, nous avons mené l'analyse d'un corpus composé de cinquante débuts de textes narratifs littéraires (romans, nouvelles, autobiographies) de langue allemande, dont la première phrase au moins est un énoncé généralisant (cette formulation signale le problème que pose la notion de début pour définir son unité : le 
début se constitue-t-il d'une phrase, d'un ensemble de phrases, d'un paragraphe, d'un ensemble de paragraphes, d'un chapitre etc.?). Les textes du corpus relèvent principalement de la fiction. Le corpus n'est pas uniquement constitué de textes contemporains.

Ont été retenus les incipits avec au moins un énoncé généralisant. Parfois une seule phrase, parfois toute une séquence généralisante. Ont été exclus les énoncés généralisants dans les titres, dans les titres de chapitre, dans les paratextes, dans la mesure où ils ne se situent pas matériellement au niveau du récit proprement dit, i.e. où ils sont explicitement présentés comme ne relevant pas de l'histoire racontée. Inversement, les énoncés généralisants en ouverture qui constituent un discours de personnage rapporté ont aussi été exclus, dans la mesure où leur appartenance au niveau de l'histoire racontée est visuellement marquée par les guillemets.

\section{Fonctions textuelles des énoncés généralisants en ouverture de textes narratifs littéraires}

30 L'analyse du corpus permet d'identifier trois fonctions principales pour les énoncés généralisants en ouverture de textes narratifs littéraires.

\section{La généralisation fournit un moule interprétatif du récit}

31 Le narrateur se présente alors comme une personne à même de prendre de la distance par rapport à l'histoire qu'elle raconte, capable d'analyse et d'interprétation, racontant une histoire en lien ou à mettre en perspective avec une vérité générale sur la vie ou la nature humaine. L'énoncé généralisant fait référence à une classe avant qu'un exemple soit introduit et présenté comme illustrant cette classe. En général cette entrée en matière permet de centrer le récit sur un personnage, même si l'énoncé généralisant évoquait une classe de situations (extraits 1 et 4).

Deux cas de figure semblent possibles: soit l'énoncé généralisant s'apparente à un prologue avant le récit à proprement parler, soit la généralisation encadre le récit, avec une reprise de l'énoncé généralisant à la fin de la nouvelle (extrait 7').

Dans la célèbre nouvelle de Stefan Zweig "Untergang eines Herzens » [Naufrage d'un cœur] (extrait 1), une entrée-préface s'étendant sur une dizaine de lignes résume la dimension universelle du récit à venir, l'ébranlement d'un individu, impuissant à se protéger, pouvant être causé par un tout petit événement. L'histoire de Salomonsohn, découvrant la sexualité de sa fille, apparaît alors comme un exemple de la disproportion entre une cause et l'effet que celle-ci peut avoir sur les hommes.

La nouvelle « Alte Pralinen » [Vieux chocolats] de Undine Gruenter (extrait 7) développe l'histoire de Madame Haussier et d'Aurélie, la fille qu'elle a recueillie. Madame Haussier raconte partout et souvent qu'Aurélie se goinfre de chocolats dans la cave. Un jour, Aurélie disparaît. Le lien existant entre les propos de Madame Haussier et la disparition d'Aurélie est fait par la narratrice: "Si Madame Haussier s'était soucié de savoir si Aurélie avait pu surprendre ses conversations au coin de la rue, elle aurait pu supposer qu'elles étaient cause de sa disparition ${ }^{31}$. C'est aussi la narratrice qui interroge les habitudes de Madame Haussier, révélées par ses propres propos : « Entasser des chocolats à la cave peut sembler bizarre $\aleph^{32}$. 

récit est encadré par la rumeur (extrait 7 '), présentée comme la cause de la disparition de la jeune fille. La rumeur est présentée par la narratrice comme l'élément explicatif de la nouvelle.

s le roman de Catalin Dorian Florescu Jacob beschließt zu lieben [Le Turbulent destin de Jacob Obertin], extrait 12, les premiers paragraphes présentent les ouragans comme un combat de Dieu contre le diable. Cette ouverture généralisante se termine sur « Ceux qui osent sortir de chez eux ces jours-là $\|^{33}$, qui implique une opération de parcours sur tous les individus osant sortir de chez eux un jour de tempête. Le focus s'arrête ensuite sur le père du narrateur et sur une tempête en particulier (de juillet 1924) présentant l'histoire à venir comme l'histoire d'un pacte avec le diable. Trois événements sont présentés comme liés au diable (le mariage du père du narrateur, la naissance du narrateur et la perte de tout) avant que ne commence le récit d'un véritable orage.

L'incipit 13 de la nouvelle de Roma Ligocka, «Die spielende Oma » [La grand-mère qui jouait] présente la mère du narrateur comme une survivante de l'Holocauste, à la suite de quoi son intérêt pour les farces et attrapes prend une autre dimension.

On le voit: avec un énoncé généralisant, le début du récit ne donne pas seulement des informations sur le contenu de l'histoire, il ne pose pas seulement de premiers éléments thématiques : il introduit d'abord une perspective narrative. Dans de nombreux cas, un narrateur omniscient présente une version subjective de son récit. L'orientation du lecteur se joue là avant tout sur le plan de la perspective narrative.

\section{La généralisation sert la captatio benevolentiae}

39 L'utilisation d'énoncés généralisants en ouverture de textes narratifs littéraires révèle aussi des narrateurs attachés à susciter le plaisir de leurs lecteurs.

Une des fonctions de l'incipit romanesque selon Andrea Del Lungo est de séduire le lecteur ${ }^{34}$. Or la séduction est, selon Philippe Breton, "une simulation de la fusion, de l'identique » : " "Je pense comme vous" dit le séducteur, "je suis comme vous" et, ajoutet-il, "vous pouvez donc penser comme moi" » ${ }^{35}$. En ce sens la généralisation, « discours de tous les sujets $»^{36}$, est bien une séduction. Et qui plus est une séduction particulièrement efficace car l'énoncé généralisant, qui, sur le plan énonciatif, n'est pas le fait de son locuteur, ne peut pas être nié. Ali Bouacha insiste sur le fait que la vérité d'un énoncé généralisant se situant au-delà du vrai ou du faux, l'interlocuteur est comme neutralisée ${ }^{37}$. Deux choses sont, pour un interlocuteur, possibles face à un énoncé généralisant : dans la mesure où il co-construit l'énoncé, il peut soit le répéter soit se taire.

4

Les ouvertures généralisantes des nouvelles de Siegfried Lenz dans son recueil So zärtlich war Suleyken [Que la vie était douce à Suleyken] (extraits 4 et 10) illustrent bien la dimension ludique attachée à ces énoncés, le plaisir que le texte cherche à susciter. Dans ces textes très largement dialogiques et contenant des adresses aux lecteurs, les énoncés généralisants sont souvent associés à des traits humoristiques. L'humour se caractérisant, comme l'a montré Béatrice Priego-Valverde, à la fois par une distance du locuteur sur son énoncé et par une complicité avec son interlocuteur ${ }^{38}$, l'énoncé généralisant tel que nous l'avons défini est particulièrement adapté à l'humour: avec l'énoncé généralisant le narrateur prend une certaine distance avec son récit et se rapproche de son lecteur, en mettant en avant leur complicité.

Cahiers de Narratologie, 31 Bis | 2017 
42 Un sujet en particulier se prête à l'ouverture généralisante et humoristique, à savoir la réflexion sur les genres littéraires, le commentaire sur la tradition littéraire, la pensée sur le lien entre monde et littérature, avec lesquels le narrateur se présente comme un lettré, qui connaît ses humanités.

43 Dans la nouvelle de Thomas Mann « Luischen » [Louisette] (extrait 14) l'ouverture sur les couples qui, dans la réalité, dépassent tout ce que la littérature ou le théâtre peuvent inventer d'associations de contraires contribue à présenter le couple formé par l'avocat Jacoby et sa femme comme un couple réaliste bien que des plus étonnants. Ici le lecteur est appelé, avec bonne volonté et en raison d'une expérience largement partagée, à croire ce qui va être raconté et à poursuivre sa lecture.

\section{La généralisation permet le partage d'une expérience personnelle difficile}

Une troisième fonction est attachée au motif de la confidence, du partage d'un vécu douloureux. L'énoncé généralisant en ouverture d'un texte narratif permet aussi au narrateur, dans un contexte de vulnérabilité et de partage d'une expérience personnelle délicate, de prendre la parole, de commencer à parler malgré tout.

Le roman de Brigitte Kronauer Frau Mühlenbeck im Gehäus [Madame Mühlenbeck dans sa cellule] (extrait 8) s'ouvre sur un souvenir d'enfance autour de la peur du noir. L'énoncé généralisant permet la prise de parole sur un partage d'expérience commune.

Dans la nouvelle de Hermann Hesse « Das erste Abenteuer » [Première aventure] (extrait 9), la première phrase du récit «J'avais dix-huit ans et je terminais mon apprentissage en mécanique $»^{39}$ est précédée de la description d'une expérience universelle sur deux paragraphes : l'oubli du vécu mais aussi le souvenir aigu, qui mobilise tous les sens. Ces deux éléments viennent justifier la prise de parole du narrateur.

Quand il y a partage d'une expérience personnelle, l'énoncé généralisant anticipe d'éventuelles interrogations sur la pertinence du récit (Pourquoi je raconte cette histoire ? Pourquoi je partage cet ou ces événement(s) personnel(s) ?) et justifie en même temps le récit par l'intérêt qu'il y a à le raconter. En outre, l'énoncé généralisant permet la prise de parole elle-même : le narrateur, qui veut et doit bien commencer à "parler ", doit dépasser ses sentiments, sa douleur ou son empathie, ce que le détour par l'expérience générale lui permet.

On notera que ces trois fonctions textuelles de l'énoncé généralisant ne sont pas exclusives l'une de l'autre : elles sont souvent présentes conjointement en texte, ce que le début de la nouvelle «Kinderseele» [Âme d'enfant] (extrait 11) de Hermann Hesse illustre parfaitement. La considération générale sur la distinction entre jours à bonnes actions et jours lourds de destin amène le narrateur à dire combien il est difficile de parler des actions relevant de la deuxième catégorie. C'est le motif de la confidence dans un contexte de vulnérabilité. L'énoncé généralisant est suivi d'un blanc sur la page. Puis le narrateur commence le récit proprement dit, avec une description du cadre géographique, à savoir la maison paternelle. Et la description qui en est fait est relativement longue, alors que l'énoncé généralisant avait "ouvert" des attentes en matière de temps ( « manchmal », « zu anderen Stunden » [parfois, à d'autres heures]) et de personnages («wir » [nous]). C'est seulement au deuxième paragraphe que celles-ci sont activées dans le récit avec « Als ich elf Jahre alt war » [À l'âge de onze ans, je], avant 
un nouveau développement au niveau du discours, où le narrateur interprète son vécu d'enfant avec les termes "Schicksal» [fatalité] et «Angst» [peur]. C'est le motif de la maturité, en même temps qu'un indice de difficulté à aborder le cœur du sujet. La prise de parole n'est possible, donc, que parce que le narrateur se présente comme un narrateur sachant partager cette expérience avec son lecteur.

\section{Narration et performance}

Les fonctions textuelles des énoncés généralisants en ouverture de textes narratifs littéraires qui ont été mises en évidence touchent à l'interprétation, la séduction, la confidence - elles sont toutes à situer au niveau de l'intersubjectivité entre le narrateur et le lecteur. Plus précisément les énoncés généralisants au début de ces textes narratifs littéraires donnent du narrateur une certaine image, i.e. ils sont pour le narrateur une manière de "présenter son moi $»^{40}$. Dans notre corpus, les narrateurs apparaissent comme disposant d'un savoir sur la nature humaine, avec une grande expérience en la matière, à même de prendre de la distance sur l'histoire qu'il raconte, particulièrement réflexif.

Nous voilà abordant la problématique de l'ethos, c'est-à-dire de l'image que le locuteur donne de lui-même à travers son discours. Spécialiste de l'ethos dans l'analyse du discours littéraire, Dominique Maingueneau s'est aussi intéressé au genre des petites annonces sur un site internet de rencontre. Il écrit à propos d'une petite annonce « imit [ant] le genre de la petite annonce traditionnelle de la presse écrite, rédigée par une agence matrimoniale » :

Ce texte met en scène un ethos "dit» qui donne une série d'informations sur le physique et le caractère de l'annonceuse. Mais à la différence de ce qui se passe en littérature ou en publicité, le lecteur est ici dans l'incapacité de construire un ethos discursif ${ }^{41}$ qui converge sémantiquement avec cet ethos « dit ». Pour dire les choses en termes plus simples, on ne perçoit pas en quoi les caractéristiques de cette énonciation montrent une locutrice [telle qu'elle se présente explicitement] ${ }^{42}$.

51 L'opposition entre le genre de la petite annonce de rencontre traditionnelle et les genres littéraires est particulièrement intéressante pour souligner qu'il est possible $d$ ' «appréhender le style comme une manière de présenter son moi » ${ }^{43}$. Dans le cas de la petite annonce évoquée, il n'y a pas de concordance entre ce que l'annonceuse dit explicitement d'elle et la manière dont elle le dit. «[I]l y a ici rupture modale entre l'énonciateur et l'énoncé $»^{44}$ : le style plat de l'annonce amène plutôt le lecteur à construire l'ethos d'une annonceuse banale. Dans le discours littéraire, ça n'est pas l'ethos dit qui est le plus courant, c'est l'ethos montré qui joue un rôle plus central.

[L'ethos montré] est construit par le destinataire à partir d'indices donnés par l'énonciation : choix d'ordre lexical, complexité de la syntaxe, rythme des phrases, jeu sur les plans énonciatifs (parenthèse, ironie, parodies...), etc., mais aussi qualité de l'orthographe, ou richesse et nature de la culture (une allusion à tel peintre italien de la Renaissance, à tel écrivain contemporain, au rock ou au rap)...5.

52 Les narrateurs de nos textes ne se disent pas tel ou tel, ils se donnent à voir tel ou tel, ils se montrent tel ou tel à travers leur mise en scène d'eux même par leur prise de parole narrative.

Par ailleurs c'est principalement la dimension « expérientielle ${ }^{46}$ de l'ethos qui est mise en jeu, à savoir la « corporalité du garant » qui permet de faire adhérer les destinataires : 
« le statut du conteur correspond à des manières de parler très différentes selon que le locuteur est un mondain qui fait son récit dans un salon ou un paysan du XIX ${ }^{\text {ème }}$ siècle qui parle à la veillée $»^{47}$.

Cette corporalité du narrateur dans nos textes littéraires est essentielle - même si elle peut paraître étonnante au premier abord. Aussi, Andrée Chauvin-Vileno rappelle « que tout genre de discours écrit doit gérer son rapport à une vocalité fondamentale $»^{48}$. Il convient selon elle de "donner même à l'écrit une très grande importance à l'actio(n) rhétorique $»^{49}$, de ne pas négliger dans les textes littéraires la "dimension de la scénographie qui articule corps et texte $»^{50}$.

Le terme "scénographie » est particulièrement intéressant pour nous : il met en avant le fait que le récit littéraire est le résultat d'un processus de narration, que le contenu narratif ou l'histoire est le fruit d'un acte narratif. Or c'est bien ce que thématisent les incipits du corpus, en activant le niveau du discours avant le niveau du récit.

Dans le même sens, Jennifer Lorenzen-Peth, dans son analyse des nouvelles de Thomas Mann, dont deux comptent parmi les textes du corpus, utilise l'adjectif " performativ $»^{51}$ [performatif] pour dire que c'est l'acte même de raconter qui constitue la perspective narrative de ces textes. Or c'est un terme utilisé au théâtre et dans les arts dramatiques quand les corps sont mis en avant sur la scène, pour eux-mêmes, sans dimension référentielle ${ }^{52}$.

57 Cela nous semble fondamental pour expliquer ce qui est en jeu dans les récits littéraires s'ouvrant sur un énoncé généralisant. En investissant pleinement le niveau de l'interaction et de l'intersubjectivité entre le narrateur et le lecteur, ceux-ci rappellent que les récits de fiction se caractérisent par un double niveau de fiction, celui de l'histoire racontée et celui de la narration : « la narration est en effet une composante de la fiction, et ses propriétés sont celles que le récit lui attribue $\aleph^{53}$. Marcel Vuillaume aussi recourt à une image tirée du théâtre : il présente le narrateur comme un «metteur en scène $»^{54}$. Nous soulignons qu'en tant que metteur en scène, le narrateur se met aussi lui-même en scène. Pour mettre en évidence le fait que le narrateur appartient au récit littéraire, Marcel Vuillaume propose de «distinguer la fiction - qui englobe l'univers narré, la narration et ses protagonistes - de l'histoire proprement dite, dont le narrateur et le lecteur ne font pas partie $\aleph^{55}$. Et de faire un parallèle avec l'art dramatique - en refusant le statut de métaphore à ce parallèle :

[L]es deux rôles [du narrateur et du lecteur] étant impliqués par le récit lui-même ou, plus exactement, par l'activité qui l'engendre, on peut se demander si la fiction marginale ne fait qu'expliciter certaines propriétés de la fiction en général ou si elle va au-delà et constitue un "supplément » de fiction, et selon G[érard] Genette ${ }^{56}$, une «transgression » [qu'il nomme metalepse]. Pour en juger, il faut essayer de saisir les œuvres de fiction dans leur spécificité en les comparant à d'autres sortes de narration. [...] [L']univers décrit dans un récit fictif n'existe pas indépendamment du discours qui le représente, de sorte que ce dernier constitue le seul mode d'accès possible à cet univers. [...] [L]es récits de fiction ne se distinguent des pièces de théâtre que par les moyens employés pour leur actualisation : celles-ci ont besoin d'une scène et d'acteurs, alors que ceux-là ne requièrent qu'un lecteur. [...] Il s'agit, me semble-t-il, d'autre chose que d'une simple métaphore, il s'agit de l'expression d'une véritable rivalité: le romancier se mesure au dramaturge et entend bien produire les mêmes effets que lui ${ }^{57}$. 


\section{Conclusion} s'ouvrant sur un énoncé généralisant rend compte de la matérialité du récit dans les textes de fiction : celui-ci est indissociable de la figure du narrateur, de sa matérialité, i.e. de sa corporalité et de la vocalité de son discours. Le récit littéraire, ça n'est pas seulement l'histoire proprement dite: le récit se compose, certes, de ce qui est narré, mais aussi de la narration, i.e. de l'acte même de narration. C'est en ce sens que nous parlons de « performance » du narrateur.

cette perspective, nous nous interrogeons sur la catégorisation des débuts narratifs. Nos analyses des débuts de textes narratifs littéraires s'ouvrant sur un énoncé généralisant tendent à montrer que les débuts métatextuels ne sont pas à mettre au même niveau que les débuts ab-ovo ou in medias res - ne serait-ce que parce que les généralisations en ouverture sont souvent suivies de débuts ab-ovo et in medias res. Par ailleurs, la présentation traditionnelle de la séquence narrative se composant de l'intrigue narrative éventuellement encadrée par une entrée-préface ou résumé et bilan ou morale nous semble aussi problématique. Les deux niveaux de fiction nous semblent beaucoup plus indissociables. Le niveau de fiction narrateur/lecteur ne nous semble pas seulement relever d'un plus possible. Il est constitutif du récit de fiction, même quand il n'est pas mis en scène.

\section{APPENDIXES}

\section{Extraits en allemand, langue originale}

1) Zu entscheidender Erschütterung eines Herzens bedarf das Schicksal nicht immer wuchtigen Ausholens und schroff verstoßender Gewalt ; gerade aus flüchtiger Ursache Vernichtung zu entfalten, reizt seine unbändige Bildnerlust. Wir nennen dies erste leise Berühren in unserer dumpfen Menschensprache Anlaß und vergleichen erstaunt sein winziges Maß mit der oft mächtig fortwirkenden Gewalt ; aber so wenig eine Krankheit mit ihrem Kenntlichwerden, so wenig beginnt das Schicksal eines Menschen erst, sobald es sichtbar und Geschehnis wird. Immer, im Geist und im Blute, waltet das Schicksal längst innen, eh es von außen die Seele berührt. Sich-Erkennen ist schon Sich-Wehren, und ein vergebliches zumeist.

Der alte Mann - Salomonsohn hieß er und durfte sich daheim Geheimer Kommissionsrat nennen - wachte nachts im Hotel von Gardone [...]. (Stefan Zweig, « Untergang eines Herzens » [1926], dans id., Verwirrung der Gefühle, Erzählungen, Francfort-sur-le-Main, 1984, p. 145-181)

2) Es gibt im Leben eine Zeit, wo es sich auffallend verlangsamt, als zögerte es weiterzugehen oder wollte seine Richtung ändern. Es mag sein, daß einem in dieser Zeit leichter ein Unglück zustößt. 
Homo besaß einen kranken kleinen Sohn ; das zog durch ein Jahr, ohne besser zu werden und ohne gefährlich zu sein, der Arzt verlangte einen langen Kuraufenthalt, und [...]. (Robert Musil, « Grigia » [1924], dans id., Drei Frauen, Reinbek bei Hambourg, 1978, p. 5-25.

3) Die geborenen Dilettanten, aus welchen ein so großer Teil der Menschheit zu bestehen scheint, könnte man als Karikaturen der Willensfreiheit bezeichnen. Indem sie nämlich die ursprüngliche Fähigkeit jedes originellen Menschen entbehren, den Ruf der Natur im eigenen Innern zu vernehmen, treiben sie leichtsinnig und unentschlossen in einem Leben scheinbarer Willkür dahin.

$\mathrm{Zu}$ diesen Vielen gehörte auch der Knabe Emil Kolb in Gerbersau, und [...]. (Hermann Hesse, « Emil Kolb » [1910], dans id., Die Erzählungen und Märchen, Berlin, 2009, p. 999-1025.

4) Oft, Herrschaften, kann schon ein kleiner Mangel Anlaß geben zu einer Reise - beispielsweise der Mangel an einem Kilochen Nägel. Von diesem Mangel betroffen fand sich in Suleyken ein Mensch namens Amadeus Loch [...]. (Siegfried Lenz, « Die Reise nach Oletzko », dans id., Erzählungen 1, 1949-1955, Hambourg, 1996, p. 299-302)

5) Seltsame Orte gibt es, seltsame Gehirne, seltsame Regionen des Geistes, hoch und ärmlich. An den Peripherien der Großstädte, dort, wo die Laternen spärlicher werden und die Gendarmen zu zweien gehen, muß man in den Häusern emporsteigen, bis es nicht weiter geht, bis in schräge Dachkammern, wo junge, bleiche Genies, Verbrecher des Traumes, mit verschränkten Armen vor sich hinbrüten, bis in billig und bedeutungsvoll geschmückte Ateliers, wo einsame, empörte und von innen verzehrte Künstler, hungrig und stolz, im Zigarettenqualm mit letzten und wüsten Idealen ringen. Hier ist das Ende, das Eis, die Reinheit und das Nichts. Hier gilt kein Vertrag, kein Zugeständnis, keine Nachsicht, kein Maß und kein Wert. Hier ist die Luft so dünn und keusch, daß die Miasmen des Lebens nicht mehr gedeihen. Hier herrscht der Trotz, die äußerste Konsequenz, das verzweifelt thronende Ich, die Freiheit, der Wahnsinn und der Tod...

Es war Karfreitag, abends um acht. Mehrere von denen, die Daniel geladen hatte, kamen zu gleicher Zeit. (Thomas Mann, « Beim Propheten » [1904], dans id., Erzählungen, Fischer (= Stockholmer Gesamtausgabe der Werke von Thomas Mann), 1960, p. 362-370)

6) Kein Land ist an Friedlichkeit jenem zu vergleichen, das in den Tod führt. Das Leben wölbt sich über dem Kopf wie ein Brückenbogen, und unten fließt das Wasser, trägt den Kahn, nimmt ihn weiter.

Friedrichsfelde bei Berlin ; ein heller Sommerabend. (Alfred Döblin, « Von der himmlischen Gnade », dans id., Die Ermordung einer Butterblume. Ausgewählte Erzählungen 1910-1950, Olten et Fribourg en Brisgau, 1962, p. 170-180)

7) In einer kleinen Stadt, in der die Hälfte der Häuser leersteht, sind Gerüchte wie alte Pralinen, die im kühlen Keller in einem Regal gestapelt und schon von einer pelzigen Schicht weißen Schimmels überzogen sind, da der Deckel der Schachtel nicht luftdicht schließt.

Im Keller eines kleinen Hauses in der Rue des Roses in Trouville saß ein Mädchen unter einer funzligen Glühbirne und aß Pralinen. (Undine Gruenter, « Alte Pralinen », dans id., Sommergäste in Trouville, Berlin, 2005 [2003], p. 112-121)

7') Es heißt, im Keller eines kleinen Hauses in der Rue des Roses in Trouville sitzt ein kleines Mädchen zwischen leer gegessenen Pralinenschachteln und blinzelt träge in die 
funzlige Birne. (Undine Gruenter, « Alte Pralinen », dans id., Sommergäste in Trouville, Berlin, 2005 [2003], p. 112-121, letzter Satz)

8) Absolute Finsternis ist etwas Grausames. Mein Vater war ein jähzorniger Mann, aber schon damals stellte er uns Kindern, wenn wir uns im Dunkeln fürchteten, ein Öllichtchen ins Schlafzimmer. Das kostete fast nichts und half so. Wenn wir nachts schrien, trug er uns singend den langen Flur auf und ab, bis wir uns beruhigt hatten. Vor nichts, vor keinem Menschen habe ich in meinem Leben Angst gehabt, nur vor dem Dunkeln. (Brigitte Kronauer, Frau Mühlenbeck im Gehäus, Stuttgart, 1980)

9) Sonderbar, wie Erlebtes einem fremd werden und entgleiten kann! Ganze Jahre, mit tausend Erlebnissen, können einem verloren gehen. Ich sehe oft Kinder in die Schule laufen und denke nicht an die eigene Schulzeit, ich sehe Gymnasiasten und weiss kaum mehr, dass ich auch einmal einer war. Ich sehe Maschinenbauer in ihre Werkstätten und windige Kommis in ihre Bureaus gehen und habe vollkommen vergessen, dass ich einst die gleichen Gänge tat, die blaue Bluse und den Schreibersrock mit glänzigen Ellenbogen trug. Ich betrachte in der Buchhandlung merkwürdige Versbüchlein von Achtzehnjährigen, im Verlag Pierson in Dresden erschienen, und ich denke nicht mehr daran, dass ich auch einmal derartige Verse gemacht habe und sogar demselben Autorenfänger auf den Leim gegangen bin.

Bis irgend einmal auf einem Spaziergang oder auf einer Eisenbahnfahrt oder in einer schlaflosen Nachtstunde ein ganzes vergessenes Stück Leben wieder da ist und grell beleuchtet wie ein Bühnenbild vor mir steht, mit allen Kleinigkeiten, mit allen Namen und Orten, Geräuschen und Gerüchen. So ging es mir vorige Nacht. Ein Erlebnis trat wieder vor mich hin, von dem ich seinerzeit ganz sicher wusste, dass ich es nie vergessen würde, und das ich doch jahrelang spurlos vergessen hatte. Ganz so wie man ein Buch oder ein Taschenmesser verliert, vermisst und dann vergisst, und eines Tages liegt es in einer Schublade zwischen altem Kram und ist wieder da und gehört einem wieder.

Ich war achtzehnjährig und am Ende meiner Lehrzeit in der Maschinenschlosserei. (Hermann Hesse, « Das erste Abenteuer » [1905], dans id., Die Erzählungen und Märchen, Berlin, 2009, p. 514-518)

10) Manchmal, wie die Erfahrung zeigt, glaubt man etwas zu besitzen, nur weil man sich an den Gedanken des Besitzes gewöhnt hat. Dieser Tatbestand war gegeben im Fall der sogenannten Suleyker Poggenwiese, eines moorigen Landzipfelchens, das erfüllt war vom quakenden Palaver der Frösche, vom einzelgängerischen Brummen der Hummeln, von unablässigem Gepieps und Gezirp. Die Suleyker, sie sahen nämliche Poggenwiese als ihren rechtmäßigen Besitz an, weshalb sie ohne Arg hinaufließen ihre berühmten Schafe, ihre Schimmel, ihre Kühe, ganz zu schweigen von den Enten, die es unaufhaltsam zu den Gräben zog.

Es ging gut, sagen wir mal - aber niemand hat die Jahre gezählt, wie lange es gut ging. Eines Tages nun zog sich ein Mensch aus Schissomir, Edmund Piepereit mit Namen, seine Schuhe aus [...]. (Siegfried Lenz, « Die große Konferenz » [1955], dans id., Die Erzählungen, Hoffmann und Campe, Hambourg, 2006, p. 304-308)

11) Manchmal handeln wir, gehen aus und ein, tun dies und das, und es ist alles leicht, unbeschwert und gleichsam unverbindlich, es könnte scheinbar alles auch anders sein. Und manchmal, zu anderen Stunden, könnte nichts anders sein, ist nichts unverbindlich 
und leicht, und jeder Atemzug, den wir tun, ist von Gewalten bestimmt und schwer von Schicksal.

Die Taten unseres Lebens, die wir die guten nennen und von denen zu erzählen uns leichtfällt, sind fast alle von jener ersten, « leichten » Art, und wir vergessen sie leicht. Andere Taten, von denen zu sprechen uns Mühe macht, vergessen wir nie mehr, sie sind gewissermaßen mehr unser als andere, und ihre Schatten fallen lang über alle Tage unseres Lebens.

Unser Vaterhaus, das groß und hell an einer hellen Straße lag, betrat man durch ein hohes Tor, und sogleich war man von Kühle, Dämmerung und steinern feuchter Luft umfangen. Eine hohe, düstere Halle nahm einen schweigsam auf, der Boden von roten Sandsteinfliesen führte leicht ansteigend gegen die Treppe, deren Beginn zuhinterst tief im Halbdunkel lag. Viele tausend Male bin ich durch dies hohe Tor eingegangen, und niemals hatte ich acht auf Tor und Flur, Fliesen und Treppe : dennoch war es immer ein Übergang in eine andere, in « unsere » Welt. Die Halle roch nach Stein [...].

Als ich elf Jahre alt war, kam ich eines Tages von der Schule her nach Hause, an einem von den Tagen, wo Schicksal in den Ecken lauert, wo leicht etwas passiert. An diesen Tagen scheint jede Unordnung und Störung der eigenen Seele sich in unserer Umwelt zu spiegeln und sie zu entstellen. Unbehagen und Angst beklemmen unser Herz, und [...]. (Hermann Hesse, « Kinderseele » [1918/19], dans id., Die Erzählungen und Märchen, Berlin, 2009, p. 1250-1277)

12) In jedem Sturm steckt ein Teufel. In einem sommerlich flüchtigen wie auch in einem, der sich tagelang schwer aufs Land legt. Er versteckt sich vor Gott. Je ängstlicher er wird, desto kräftiger wirbelt er die Luft und die Erde auf. Doch auch das nützt ihm wenig. Wenn dann der Sturm draußen auf den Feldern jault, wissen die Menschen, dass Gott den Teufel gefunden hat.

Hat er Glück, so kann er fliehen. Er tritt aus dem Orkan heraus, der Wind legt sich, und die Wolken lösen sich auf, als ob es sie nie gegeben hätte. Aber es ist zu früh zum Aufatmen, zu dringend braucht der Gehetzte neue Tarnung. Er wird sie im Fell einer Katze oder in der dichten Krone einer Buche suchen. Wer sich an solchen Tagen aus dem Haus traut, rafft die Kleider fester um den Körper, damit der Teufel sich nicht einschleicht.

Im Juli 1924 kam mein Vater aus solch einem Gewitter heraus, und er widersprach jenen nie, die meinten, er habe mit dem Teufel paktiert. Nicht, als er Mutter heiratete, nicht als sie mich bekamen, und auch nicht, als er alles wieder verlor.

Als damals die Wolkenfront im Westen, noch hinter der Grenze zu Ungarn, sich bedrohlich vorwärtsschob, sprang der alte Feldwächter auf. Der Donner hatte ihn geweckt [...]. (Catalin Dorian Florescu, Jacob beschließt zu lieben, Munich, 2012 [2011])

13) Eine richtige Lebensfreude empfinden die Überlebenden des Holocaust selten. Oder sie erleben sie anders als die anderen. Unbeschwerte, sprühende Fröhlichkeit, Ausgelassensein, lautes Lachen stellen sich nicht ein. Wenn dergleichen doch durchbricht - denn das Leben ist oft stärker als der Schmerz -, dann ist es, als würde sich sofort eine kalte Hand der Erinnerung auf die Brust des Lachenden legen oder ihm über den Mund fahren. Aber wie alle Menschen brauchen auch die Überlebenden des Holocaust Freude am Leben, und manchmal, für kurze Momente wird sie ihnen auch zuteil. 
Bei meiner Mutter war es so, als sie die Scherzartikel entdeckte.

Ihr Enkel Jakob war damals im richtigen Spielalter, und in dem Geschäft um die Ecke wurden damals Scherzartikel eingeführt. Die Oma und ihr Enkel kauften also haufenweise Knallfrösche [...] (Roma Ligocka, « Die spielende Oma », dans id., Ein Lächeln, eine Rose, Munich, 2001, p. 37-43)

14) Es gibt Ehen, deren Entstehung die belletristisch geübteste Phantasie sich nicht vorzustellen vermag. Man muß sie hinnehmen, wie man im Theater die abenteuerlichen Verbindungen von Gegensätzen wie Alt und Stupide mit Schön und Lebhaft hinnimmt, die als Voraussetzung gegeben sind und die Grundlage für den mathematischen Aufbau einer Posse bilden.

Was die Gattin des Rechtsanwalts Jacoby betrifft, so war sie jung und schön, eine Frau von ungewöhnlichen Reizen. Vor - sagen wir einmal - dreißig Jahren war sie auf die Namen Anna, Margarethe, Rosa, Amalie getauft worden, aber man hatte sie [...] (Thomas Mann, « Luischen » [1903], dans id., Frühe Erzählungen, Francfort sur le Main, 1981, p. 153-171)

\section{Extraits, traduction française}

1) Pour ébranler un cœur de manière décisive, le destin n'a pas nécessairement recours à un bras armé ou à la force brutale ; au contraire, engendrer la destruction à partir d'un motif futile, voilà une stimulation à son irrépressible talent créatif. Dans l'approximation de notre langage humain, nous nommons ce discret contact initial « prétexte » et nous sommes surpris quand nous comparons l'insignifiance de ce dernier avec les conséquences souvent dramatiques qu'il déchaîne à sa suite ; mais, de même qu'une maladie n'a pas débuté juste au moment où l'on en prend conscience, de même le destin d'un homme ne se joue-t-il pas simplement à partir de l'instant où il devient tangible. Toujours, dans l'esprit et dans le sang, le destin s'organise de l'intérieur, à l'abri des regards, bien avant que l'âme ne soit touchée de l'extérieur. Se connaître soi-même, c'est déjà se défendre - et c'est le plus souvent une cause perdue.

Le vieil homme - il s'appelait Salomonsohn et s'honorait dans son pays du titre de Geheimer Kommissionsrat - se réveilla durant la nuit dans cet hôtel de Gardone [...]. (Stefan Zweig, « Naufrage d'un cœur » [1926], traduit par Irène Kuhn, dans id., La Confusion des sentiments et autres récits, éd. par Pierre Deshusses, Paris, 2013, p. 881-911)

2) Pour tout homme, à une certaine période, la vie ralentit visiblement, comme si elle hésitait à continuer ou songeait à changer de direction. C'est dans cette période-là, peutêtre, que les accidents arrivent le plus facilement.

Homo avait un fils malade : cela traîna un an sans s'améliorer ni s'aggraver. Le médecin prescrivit une longue cure, et [...] (Robert Musil, « Grigia » [1924], dans id., Trois femmes suivi de Noces, traduit par Philippe Jaccottet, Paris, 1962, p. 9-39)

3) On pourrait dire des amateurs-nés qui constituent, semble-t-il, la majeure partie de l'humanité, qu'ils sont des caricatures du libre-arbitre ; c'est-à-dire qu'étant dépourvus de l'aptitude élémentaire de tout être original à percevoir en lui-même l'appel de la nature, ils végètent, irrésolus et insouciants, au gré d'une existence apparemment arbitraire.

Un garçon de Gerbesau, Émile Kolb, faisait partie, lui aussi, de cette multitude, et [...]. (Hermann Hesse, « Emil Kolb » [1910], dans id., Berthold : nouvelles, traduit par Edmond Beaujon, Paris, 1977, p. 109-148) 
4) Souvent, Mesdames et Messieurs, même une petite pénurie peut devenir l'occasion d'un voyage - par exemple la pénurie d'un petit kilo de clous. Ce genre de pénurie vint à toucher un homme à Suleyken qui s'appelait Amadeus Loch [...]. (Siegfried Lenz, « Le voyage à Oletzko ", notre traduction $\mathrm{ALDC}^{58}$ )

5) Il est des lieux étranges, des cerveaux étranges, d'étranges régions de l'esprit, hautes et indigentes. À la périphérie des grandes villes, là où les réverbères se font plus rares et où les gendarmes vont par deux, il faut grimper jusqu'au dernier palier des maisons, jusqu'à des mansardes déclives où de jeunes génies hâves, des criminels du rêve, les bras croisés, ruminent leurs pensées ; jusqu'à des ateliers loués à bon marché et décorés de façon suggestive où des artistes solitaires, révoltés et consumés du dedans, fiers et affamés, enveloppés dans la grisante fumée des cigarettes, sont aux prises avec des idéaux suprêmes et échevelés. Là, c'est la fin, la glace, la pureté, le néant. Là, plus aucune convention ne compte, ni concession, ni scrupule, ni mesure, ni valeur. L'atmosphère y est chaste, si raréfiée que les miasmes de la vie n'y peuvent que prospérer. C'est le royaume du défi, de la conséquence poussée à l'extrême, où le Moi trône désespérément, le royaume de la liberté, de la folie et de la mort.

C'était un vendredi saint, le soir, vers huit heures. Plusieurs des invités de Daniel arrivèrent en même temps. (Thomas Mann, « Chez le prophète » [1904], traduit par Louise Servicen, dans id., Romans et nouvelles II, 1904-1924, Paris, 1995 (Classiques Modernes), p. 13-21)

6) Aucun pays ne ressemble autant en tranquillité à celui qui conduit à la mort. La vie se voûte au-dessus de la tête, comme l'arche d'un pont, et en dessous coule l'eau qui porte la barque et l'emporte au loin.

Friedrichsfelde près de Berlin par une claire soirée d'été. (Alfred Döblin, « De la grâce céleste », notre traduction ALDC)

7) Dans une petite ville où la moitié des maisons est à l'abandon, les rumeurs sont comme de vieux chocolats empilés sur une étagère au frais à la cave et déjà recouverts d'une couche velue de moisissure blanche en l'absence de couvercle hermétique.

Dans la cave d'une petite maison de la rue des roses à Trouvillle, une petite fille était assise dans la faible clarté d'une ampoule et mangeait des chocolats. (Undine Gruenter, « Vieux chocolats », notre traduction ALDC)

7') On raconte que dans la cave d'une petite maison de la rue des roses à Trouville, une petite fille est assise au milieu de boîtes de chocolats qu'elle a mangés, clignant faiblement des yeux dans la faible clarté d'une ampoule. (Undine Gruenter, «Vieux chocolats », dernière phrase, notre traduction ALDC)

8) L'obscurité absolue est une chose horrible. Mon père était un homme colérique, mais déjà à l'époque, quand, petits, nous avions peur du noir, il nous portait une petite lampe à huile dans la chambre. C'était efficace et cela ne coûtait rien. Quand nous pleurions la nuit, il nous prenait dans ses bras et nous promenait en chantant dans le couloir, jusqu'à ce que nous nous soyons calmés. Dans la vie, je n'ai jamais eu peur de rien ni de personne, à l'exception du noir. (Brigitte Kronauer, Madame Mühlenbeck dans sa cellule, notre traduction ALDC)

9) C'est curieux comme ce qu'on a vécu peut nous devenir étranger et nous échapper ! Des années entières, faites de mille événements, peuvent s'effacer de notre mémoire. Je vois 
souvent des enfants courant sur le chemin de l'école sans penser à ma propre scolarité, je vois des collégiens et me rappelle à peine en avoir été un moi aussi. Je vois des constructeurs de machines se rendre dans leurs ateliers et des commis pressés rejoindre leurs bureaux et j'ai complètement oublié qu'à une époque j'empruntais ces mêmes parcours, portais la blouse bleue et celle de comptable aux coudes brillants. Je considère dans la librairie d'étonnants carnets de rimes de jeunes gens de dix-huit ans, parus chez Pierson à Dresde, sans jamais penser que moi aussi autrefois j'ai écrit des vers semblables, pris au piège du même chasseur d'auteurs.

Jusqu'à ce que soudain, au cours d'une promenade, d'un voyage en train, ou à l'occasion d'une insomnie, tout un morceau de vie oublié fasse retour, qui se retrouve devant moi, éclairé crûment comme sur une scène, avec maints détails, avec tous les noms et les lieux, les bruits et les odeurs. C'est ce qui m'arriva la nuit dernière. Un événement me revint, dont j'avais la certitude à l'époque que je ne l'oublierai jamais et que j'avais pourtant oublié pendant des années sans la moindre trace. Tout comme on égare un livre ou un couteau suisse, qu'on cherche d'abord puis qu'on oublie, pour le retrouver un jour dans un tiroir au milieu de vieilleries, il est là et nous appartient à nouveau.

J'avais dix-huit ans et je terminais mon apprentissage en mécanique. (Hermann Hesse, «La première aventure ", notre traduction ALDC)

10) Parfois, comme le montre l'expérience, on croit posséder une chose simplement parce qu'on s'est habitué à l'idée de sa possession. Cet état de fait se trouvait vérifié dans le cas d'un pré dit Poggenwiese à Suleyken, une langue de terre marécageuse, résonnant du coassement bavard des grenouilles, du vrombissement solitaire des bourdons, et autres chants et sifflements incessants. Les habitants de Suleyken, eux, considéraient ce pré comme leur propriété légitime, raison pour laquelle ils ne voyaient pas de mal à y emmener paître leurs célèbres moutons, leurs chevaux blancs, leurs vaches, sans parler des canards irrésistiblement attirés par les fossés

Tout allait bien, dirons-nous - mais personne n'a compté les années pendant lesquelles tout allait bien. Et voilà qu'un jour un habitant de Schissomir, dénommé Edmund Piepereit, retira ses chaussures [...]. (Siegfried Lenz, « La grande conférence », notre traduction ALDC)

11) Parfois nous agissons, nous allons et venons, faisons ci et ça, et tout paraît facile, aisé et anodin, et toute chose pourrait très bien être autre. Et parfois, à d'autres heures, tout est au contraire irréversible, contraignant et sans légèreté, la moindre respiration est le jouet de la fatalité et lourde de conséquences.

Les actions de notre vie, que nous considérons comme bonnes et dont nous parlons aisément, sont presque tous du premier type, «faciles », et nous les oublions facilement. En revanche, il est d'autres actions, que nous évoquons avec difficulté et que nous n'oublierons jamais, c'est comme si elles nous appartenaient plus que les autres, et leurs ombres planent sur tous les jours de notre vie.

L'accès à notre maison [...] se faisait par la maison familiale, grande et claire au bord d'une route claire, par une porte cochère, et on était aussitôt plongé dans la fraîcheur, l'obscurité et le parfum humide des pierres. Un grand hall sombre accueillait silencieusement le visiteur, le sol carrelé de grès rouge montait en légère pente vers l'escalier, qui commençait tout au fond dans une épaisse pénombre. Je suis entré plusieurs milliers de fois par cette porte cochère et n'ai jamais prêté attention à la porte 
et au couloir, au sol et à l'escalier : pourtant c'était à chaque fois le passage dans un autre monde, dans « notre » monde. Le hall sentait la pierre [...].

À l'âge de onze ans, je rentrai un jour après l'école à la maison, et c'était un de ces jours, où la fatalité guette dans les coins, où facilement un événement se produit. Ces jours-là, le moindre désordre et trouble de notre âme semble se refléter alentour et imprégner notre environnement. Le malaise et la peur oppressent notre cœur, et [...]. (Hermann Hesse, « Âme d'enfant », notre traduction ALDC)

12) Toute tempête renferme un diable. Aussi bien les tempêtes d'été passagères que celles qui s'abattent sur le pays pendant plusieurs jours. Le diable se cache de Dieu. Plus il a peur, plus il soulève la terre et les airs avec force. Mais cela ne lui sert pas à grand chose. Quand la tempête hurle à travers champs, les hommes savent déjà que Dieu a trouvé le diable.

S'il a de la chance, il arrive à s'enfuir. Il sort de l'ouragan, le vent tombe et les nuages se dissipent comme s'ils n'avaient jamais existé. Mais il est trop tôt pour respirer, car le diable traqué a urgemment besoin d'un nouveau camouflage. Il le cherche dans la fourrure d'un chat ou dans l'épaisse cîme d'un hêtre. Ceux qui osent sortir de chez eux ces jours-là resserrent leurs vêtements plus près du corps pour qu'il ne s'y faufile pas.

Mon père surgit d'un tel orage en juillet 1924 et en contredit jamais ceux qui pensaient qu'il avait pactisé avec le diable. Ni le jour où il épousa ma mère, ni lorsqu'elle me mit au monde, ni lorsqu'il perdit tous ses biens.

Quand le front nuageux qui était encore derrière la frontière hongroise, à l'ouest, se mit à avancer dangereusement, le vieux garde champêtre se leva d'un bond. Le tonnerre l'avait réveillé [...]. (Catalin Dorian Florescu, Le turbulent destin de Jacob Obertin [2011], traduit par Barbara Fontaine, Paris, 2013, p. 7).

13) Il est rare que les survivants de l'Holocauste éprouvent une réelle joie de vivre. Ou alors ils la ressentent autrement que les autres. Ils ne connaissent pas la gaieté insouciante et pétillante, l'exubérance, les rires bruyants. S'il arrive malgré tout que de tels sentiments affleurent - car la vie est souvent plus forte que la douleur -, alors c'est comme si la main froide du souvenir se posait aussitôt sur la poitrine du rieur ou lui fermait la bouche. Pourtant, comme tout un chacun, les survivants de l'Holocauste ont eux aussi besoin de joie de vivre, et ils y parviennent parfois pour de courts instants.

Pour ma mère, ce fut le cas quand elle découvrit les farces et attrapes.

Son petit-fils Jakob était alors en âge d'y jouer, et la boutique au coin de la rue venait de recevoir des articles de farce et attrape. Si bien que la grand-mère et son petit-fils s'achetèrent quantité de pétards [...]. (Roma Ligocka, « La grand-mère qui jouait », notre traduction ALDC)

14) Il est des mariages que l'imagination la plus familiarisée avec la littérature ne saurait se représenter. Il les faut admettre, comme on admet au théâtre l'alliage des contrastes les plus abracadabrants, notamment celui du barbon stupide avec une femme belle et vive, combinaison et postulat qui servent de base à l'édification mathématique d'un vaudeville.

L'épouse de l'avoué Jacoby était jeune et belle, douée d'un charme rare. Environ - mettons - trente ans auparavant, elle avait reçu sur les fonts baptismaux les prénoms d'Anna Marguerite Rose Amélie ; mais [...] (Thomas Mann, « Louisette » [1903], traduit par 
Louise Servicen, dans id., Romans et nouvelles I, 1896-1903, Paris, 1994 (Classiques

Modernes), p. 95-110)

\section{NOTES}

1. Au-delà de sa dimension esthétique, le texte narratif littéraire est souvent donné comme exemple prototypique du texte cohérent ou du texte empreint d'intertextualité.

2. Robert Beaugrande et Wolfgang Dressler, Einführung in die Textlinguistik, Tübingen, de Gruyter, 1981. Les sept critères de textualité qu'ils définissent sont la cohésion, la cohérence, l'intentionnalité, l'acceptabilité, l'informativité, la situationnalité et l'intertextualité. Il ne s'agit pas de critères nécessaires et suffisants, mais de dimensions servant à la description des caractéristiques essentielles de textes (prototypiques), comme le souligne Kirsten Adamzik, Textlinguistik. Eine einführende Darstellung, Tübingen, 2004, p. 53.

3. Ulla Fix, "Nichtsprachliches als Textfaktor: Medialität, Materialität, Lokalität », dans Zeitschrift für Germanistische Linguistik, nº 36/3, 2008, p. 343-354.

4. Voir Nobert Richard Wolf, «Texte als Bilder», dans : Ulla Fix et Hans Wellmann (éd.), Bild im Text - Text im Bild, Heidelberg, Winter, 2000, p. 289-305, ici p. 289 : « Texte werden zuvörderst mit den Augen wahr- und aufgenommen; Texte wirken mithin wie Bilder wenn nicht gar als Bilder. »

5. Martin Steinseifer, «Texte sehen. Diagrammatologische Impulse für die Textlinguistik », dans Zeitschrift für Germanistische Linguistik, ${ }^{\circ}$ 41/1, 2013, p. 8-39.

6. Nous traduisons ainsi "Sehfläche », terme utilisé par Ulrich Schmitz, "Sehflächenforschung. Eine Einführung», dans Hajo Diekmannshenke, Michaël Klemm et Hartmut Stöckl (éd.), Bildlinguistik. Theorien - Methoden - Fallbeispiele, Berlin, Erich Schmidt, 2011, p. 23-42.

7. Jean-Michel Adam, La linguistique textuelle. Introduction à l'analyse textuelle des discours. $2^{\text {ème édition }}$ , Paris, Armand Colin, 2008, p. 27 et suivantes.

8. id., Eléments de linguistique textuelle : théorie et pratique de l'analyse, Paris, Mardaga, 1990, p. 68-69. 9. Il ne nous est pas possible ici d'évoquer les débats sur la définition du "texte». Il est important en revanche de signaler que nous retenons une acception de "texte" au sens de résultat d'intention de communication. Voir Beaugrande/Dressler, 1981 (note 2). Cette définition implique de considérer aussi des genres de texte oraux.

10. Fix, 2008 (note 3), p. 347.

11. Harmut Stöckl, « Typographie : Gewand und Körper des Textes - Linguistische Überlegungen zu typographischer Gestaltung ", dans Zeitschrift für angewandte Linguistik, n 41, 2004, p. 5-48, p. 43, cité d'après Fix, 2008 (note 3), p. 348.

12. Nous pensons que tout texte a un début et une fin. Voir les critères de textualité définis par Jean-Michel Adam, «Qu'est-ce qu'un texte ?», dans séminaire MODYCO, séance du 19 mars 2013, conférence en Sorbonne.

13. William Labov et Joshua Waletzky, « Narrative analysis : oral versions of personal experience ", dans June Helm (éd.), Essays on the verbal and visual arts, Seattle, University of Washington Press, 1967, p. 12-44.

14. Jean-Michel Adam, Les Textes : types et prototypes, Paris, Armand Colin, 2011.

15. id., Le Texte narratif. Précis d'analyse textuelle (avec des travaux d'application et leurs corrigés), Paris, Fernand Nathan, 1985, p. 160.

16. Annette Retsch, Paratext und Textanfang, Würzburg, Königshausen u. Neumann, 2000 (Würzburger Beiträge zur deutschen Philologie, t. XVIII), p. 140.

17. ibid., p. 143: "Der "metatextuelle Anfang" ist meines Erachtens auch als Folge der Entwicklung des Paratextes zu sehen. Da im xIx. Jahrhundert das Verfassen von Vorworten an 
Popularität verliert, wählen Schriftsteller wie Raabe und Frenssen am Ende des XIX. und Beginn des xx. Jahrhunderts den für den Textanfang prädestinierten Ort, um bestimmte Wirkungsabsichten mitzuteilen. » (Les citations en allemand sont traduites en français par nos soins, sauf quand le nom d'un traducteur est expressément mentionné).

18. Voir Anne-Laure Daux-Combaudon, «Verallgemeinernde Äußerungen zwischen Verständnissicherung und Unterhaltsamkeit », dans Anne Larrory (éd.), Intersubjektivität und Sprache. Zu Angleichungsphänomenen in der Kommunikation, Tübingen, Narr, 2012 (Eurogermanistik, t. xxx), p. 67-89.

19. Jean-Christophe Pellat, «Les Maximes de La Rochefoucauld: formes générales d'un discours particulier », dans Franck Neveu (éd.), Faits de langue et sens des textes, Paris, SEDES, 1998, p. 111.

20. Sur la notion d'ajustement, voir Anne Larrory, « Einleitung », dans id. (éd.), 2012 (note 18), p. VII-XV.

21. Anne-Laure Daux-Combaudon, «Verallgemeinernde Äußerungen als Indizien von Dialogizität », dans Zofia Berdychowska, Joanna Janicke et Agnieszka Vogelgesang-Doncer (éd.), Texte - Textsorten - Phänomene im Text, Francfort sur le Main, Peter Lang, 2014 (Studien zur Textund Diskursforschung, t. vII), p. 27-42.

22. Magid Ali Bouacha, «Enonciation, argumentation et discours : le cas de la généralisation », dans Semen, n08, 1993. URL : http://semen.revues.org/3985?lang=en, page consultée le 28 avril 2016.

23. Dans la théorie de l'énonciation d'Antoine Culioli, l'opération de parcours est une opération lors de laquelle toutes les occurrences d'une classe sont considérées, c'est-à-dire que toutes les positions sémantiques de cette classe sont parcourues sans que le parcours ne s'arrête sur une en particulier. Voir Antoine Culioli, Pour une linguistique de l'énonciation - Opérations et représentations. Tome 1, Paris, Ophrys, 1990.

24. Jean-Claude Anscombre, "Le ON-locuteur: une entité aux multiples visages », dans Jaques Bres et al. (éd.), Dialogisme et polyphonie. Approches linguistiques, Bruxelles, Duculot, 2005 (Champs linguistiques), p. 75-94.

25. Notion introduite par Alain Berrendonner, Eléments de pragmatique linguistique, Paris, Minuit, 1981.

26. Anscombre, 2005 (note 24).

27. Daux-Combaudon, 2012 (note 18), p. 70-71.

28. ibid., p. 71-73.

29. Ali Bouacha, 1993 (note 22), p. 10-11.

30. Avec ein, «c'est par l'intermédiaire d'une occurrence quelconque que l'on fait référence à l'ensemble de la classe»; avec die, "[les différentes occurrences de la classe] étant toutes identifiées à l'occurrence type, [elles] n'apparaissent alors plus dans leur singularité ", mais elles sont toutes envisagées, comme l'indique Éric Gilbert, "La théorie des Opérations Énonciatives d'Antoine Culioli ", dans : Pierre Cotte et al. (éd.), Les théories de la grammaire anglaise en France, Paris, Hachette, 1993, p. 78-94, ici p. 90-91, cité d'après Gérard Mélis, «Peut-on différencier l'opération de parcours ? ", dans Corela, numéro spécial « Le parcours », 2006. Accessible en ligne à l'URL: http://09.edel.univ-poitiers.fr/corela/index.php?id=1401, page consultée le 28 avril 2016. Cette différence s'exprime aussi en d'autres termes : "Alors que l'indéfini "un" permet de renvoyer à un objet non spécifique mais conforme au type (un cheval est craintif), le défini singulier "le" renvoie à l'objet typique désigné par le reste du GN, le pluriel générique de l'article défini invite à recherche l'ensemble maximal des objets désignables par une telle expression » (Jean-Christophe Pellat, 1997 (note 19), p. 100).

31. "Hätte Madame Haussier sich etwas daraus gemacht, dass Aurélie die Gespräche an der Straßenecke mitangehört haben könnte, hätte sie vielleicht darin den Grund für ihr Verschwinden vermutet. » (notre traduction ALDC) 
32. «Es mag seltsam scheinen, dass Pralinen im Keller gestapelt werden. " (notre traduction ALDC)

33. « Wer sich an solchen Tagen aus dem Haus traut. » (notre traduction ALDC)

34. Andrea Del Lungo, L'incipit romanesque, Paris, Seuil, 2003 (Poétique). Outre la séduction, il définit quatre fonctions pour l'incipit romanesque : codification, thématisation, information, dramatisation.

35. Philippe Breton, «Le langage de la séduction en politique», 2007. URL: http:// argumentation.blog.lemonde.fr/2007/01/07/le-langage-de-la-seduction-en-politique/, page consultée le 7 janvier 2009.

36. Ali Bouacha, 1993 (note 22), p. 7.

37. id., «La question générique : statut linguistique et enjeu discursif », dans Sophie Moirand et al. (éd.), Parcours linguistiques de discours spécialisés, Bern, Peter Lang, 1994 (Sciences pour la communication, $\left.{ }^{\circ} 41\right)$, p. 279-289, ici p. 283.

38. Béatrice Priego-Valverde, L'humour dans la conversation familière: description et analyse linguistiques, Paris, Harmattan, 2003.

39. « Ich war achtzehnjährig und am Ende meiner Lehrzeit in der Maschinenschlosserei. » (notre traduction ALDC)

40. Dominique Maingueneau, «De l'ethos au style: la représentation de soi sur les sites de rencontre ", dans Eric Bordas / Georges Molinié (éd), Style, langue et société, Paris, Honoré Champion, 2015, p. 283-297, ici p. 284.

41. L'ethos est "discursif » lorsqu'il est un effet de l'énonciation en cours, par différence avec l'ethos " préalable ", dont on dispose avant la prise de parole de l'énonciateur, par exemple dans le cas de personnalités médiatiques. Voir Dominique Maingueneau, «Le recours à l'ethos dans l'analyse du discours littéraire ", dans Fabula, colloques en ligne « Posture d'auteurs : du Moyen Âge à la modernité ", 2014. URL: http://www.fabula.org/colloques/document2424.php, page consultée le 12 janvier 2017.

42. Maingueneau, 2015 (note 40), p. 288-289.

43. ibid., p. 284.

44. ibid., p. 289.

45. ibid., p. 287.

46. Pour la différence avec les dimensions catégorielle et idéologique de l'ethos, voir Maingueneau, 2014 (note 41).

47. Maingueneau, 2014 (note 41).

48. Andrée Chauvin-Vileno, «Ethos et texte littéraire. Vers une problématique de la voix », dans : Semen, $n^{\circ} 14,2002$ (l'auteur souligne). URL : https://semen.revues.org/2509, page consultée le 12 janvier 2017. Elle cite ici Dominique Maingueneau, Le contexte de l'œuvre littéraire. Enonciation, écrivain, société, Paris, Dunod, 1993, p. 139.

49. ibid.

50. ibid.

51. Jennifer Lorenzen-Peth, Erzählperspektive und Selbstreflexion in Thomas Manns Erzählungen. Sinnkonstitution und Sinndestruktion, Kiel, Ludwig, 2008, p. 344.

52. Voir par exemple Erika Fischer-Lichte qui définit l'acte performatif («performatiker Akt») comme une "action corporelle» («körperliche Handlung») : Erika Fischer-Lichte, Ästhetik des Performativen, Francfort-sur-le-Main, Suhrkamp, 2004, p. 37.

53. Marcel Vuillaume, Grammaire temporelle des récits, Paris, Minuit, 1990, p. 59.

54. ibid., p. 71.

55. ibid., p. 110 (l'auteur souligne).

56. Gérard Genette, Figures III, Paris, Seuil, 1972, p. 243-246.

57. Vuillaume, 1990 (note 53), p. 110-111. 
58. J'ai plaisir à remercier Sarah Neelsen pour sa relecture des traductions et pour ses suggestions pertinentes.

\section{ABSTRACTS}

Cet article s'intéresse, dans un corpus de romans et nouvelles en langue allemande s'ouvrant sur un énoncé généralisant, à la notion d'espace du texte. Pris dans sa matérialité, comme surface visuelle, le texte littéraire s'imprime en noir sur la page blanche. Pourtant, lorsqu'il commence par un énoncé généralisant, l'histoire proprement dite ne semble commencer qu'après, avec souvent des " débuts » caractéristiques d'un incipit, introduisant les éléments attendus dans la phase d'orientation-exposition (informations sur le lieu, le temps, un personnage, éventuellement plongée in medias res). L'analyse du début matériel du texte, i.e. des fonctions textuelles des énoncés généralisants dans le corpus, met en évidence que le récit commence avant l'histoire : ces débuts métatextuels mettent en jeu l'intersubjectivité entre le narrateur et le lecteur. Avec eux est investi le niveau de l'interaction entre le narrateur et le lecteur, ils assoient la prise de parole narrative. Au-delà, ils « mettent en scène » la corporalité du narrateur, la vocalité de son discours. Ces incipits illustrent donc le fait que la performance, i.e. la narration, est constitutive du récit de fiction.

In diesem Artikel, der sich mit dem Begriff des Textraums befasst, werden Anfänge von Romanen und Novellen in deutscher Sprache analysiert, die mit einer verallgemeinernden Äußerung beginnen. Wenn man den literarischen Text in seiner Materialität bzw. als Sehfläche betrachtet, erscheint vor allem das Schwarze auf der weißen Seite. Wenn nun der Text mit einer verallgemeinernden Äußerung anfängt, scheint die Erzählung erst danach zu beginnen, und zwar häufig mit für ein Incipit typischen « Änfängen », die die in der Orientierungsphase traditionell erwarteten Elemente einführen (Informationen über Ort, Zeit, Protagonisten, eventuell auch Eintauchen in die Handlung). Die Analyse des materiellen Anfangs des Textes, bzw. der textuellen Funktionen von verallgemeinernden Äußerungen im Korpus, macht deutlich, dass die Erzählung vor der eigentlichen Geschichte anfängt: unsere metatextuellen Anfänge bringen die Intersubjektivität zwischen Erzähler und Leser in den Vordergrund. Mit ihnen kann das Niveau der Interaktion zwischen Erzähler und Leser investiert werden. Indem er das Wort ergreift, setzt sich der Erzähler durch. Darüber hinaus setzt die verallgemeinernde Äußerung die Körperlichkeit des Erzählers in Szene, i.e. die Vokalität seines Diskurses. Die Incipits des Korpus veranschaulichen also die Tatsache, dass das Erzählen und die Performanz Bestandteile der fiktionalen Erzählung sind.

\section{INDEX}

Mots-clés: linguistique textuelle, matérialité, énoncés généralisants, incipit, début de texte Geographical index: Allemagne

Chronological index: XXème siècle, XXIème siècle 


\section{AUTHOR}

\section{ANNE-LAURE DAUX-COMBAUDON}

Anne-Laure Daux-Combaudon a fait des études de germanistique et de lettres modernes. Agrégée d'allemand, elle est maître de conférences en linguistique allemande à l'Université Sorbonne Nouvelle - Paris 3 et membre du Centre d'études et de recherches sur l'espace germanophone (CEREG, EA 4223). Elle enseigne la langue allemande (grammaire, textes de spécialité, langue des affaires) au Département de Langues étrangères appliquées. Ses travaux et ses publications portent sur l'approche linguistique des genres textuels. Elle s'intéresse notamment aux débuts de texte. 